\title{
MUSTIKA PAAJAH-AJAHAN PAWATEKAN SAJERONING SATUA-SATUA BANYOL RING BUKU KASUSASTRAAN BALI BALAI PENELITIAN BAHASA
}

\author{
Putu Suryaning Arum ${ }^{1}$, I. B. Putra Manik Aryana ${ }^{1}$, I. B. Rai ${ }^{2}$ \\ Jurusan Pendidikan Bahasa Bali \\ Universitas Pendidikan Ganesha \\ Singaraja, Indonesia \\ e-mail: \{yukajashienz@yahoo.com, manik.aryana, \\ bagus.rai\}@undiksha.ac.id
}

\begin{abstract}
KUUB
Tetilikan matetujon nlatarang: (1) wangun ring jero kria sastra, (2) mustika paajahajahan pawatekan, lan (3) pikenoh. Wit data sajeroning tetilikan puniki inggih punika satua-satua banyol ring buku Kasusastraan Bali Balai Penelitian Bahasa warsa 1976, sane kapupulang olih I Gusti Ngurah Bagus. Tetilikan nganggen kramaning dokumentasi ngwacen lan nyatet ri kala mupulang data wangun ring jero kria sastra, mustika paajah-ajahan pawatekan, lan pikenoh. Data tureksa sane kanggen: (1) reduksi data; (2) nlatarang data; lan (3) panyutetan (verifikasi). Pikolih tetilikan satua-satua banyol ring buku kasusastraan Bali Balai Penelitian Bahasa, sakadi: (1) wangun ring jero kria sastra wenten pitu, sakadi: murda, unteng, pragina, rerawatan, lelintihan, panampen jana, lan piteket. (2) Mustika paajahajahan pawatekan kapolihang tigalas, sakadi: sradha bhakti, arjawa, awiwahara, tinut sesana, teleb makarya, gina, sida ngraga, rasa meled uning, masawitra, metri, ulati pawongan, ulati palemahan, lan tinut swadharma. (3) Pikenohnyane prasida kaanggen sesuluh sajeroning kahuripan jana.
\end{abstract}

Kruna jejaton: Mustika Paajah-ajahan Pawatekan, Satua Banyol

\section{ABSTRAK}

Penelitian ini bertujuan menjelaskan: (1) unsur intrinsik; (2) nilai pendidikan karakter bangsa; dan (3) manfaat nilai pendidikan karakter bangsa. Sumber data dalam penelitian ini adalah cerita-cerita lucu yang ada dalam buku Kasusastraan Bali Balai Penelitian Bahasa tahun 1976, yang dikumpulkan oleh I Gusti Ngurah Bagus. Penelitian ini menggunakan metode dokumentasi teknik baca catat pada saat mengumpulkan data unsur intrinsik, nilai pendidikan karakter bangsa, dan manfaat nilai pendidikan karakter bangsa. Analisis data yang digunakan: (1) reduksi data; (2) deskripsi data; dan (3) kesimpulan/verifikasi. Hasil penelitian cerita-cerita lucu di buku kesusastraan Bali Balai Penelitian Bahasa, antara lain: (1) unsur intrinsik ada tujuh, seperti: judul, tema, tokoh, setting latar, alur, sudut pandang, dan amanat, (2) nilai pendidikan karakter bangsa ditemukan delapan belas, seperti: religius, jujur, toleransi, disiplin, kerja keras, kreatif, mandiri, rasa ingin tahu, bersahabat, cinta damai, peduli sosial, peduli lingkungan, dan tanggung 
jawab, (3) bermanfaat sebagai cerminan bertingkah laku yang baik dalam kehidupan.

Kata kunci: Pendidikan Karakter Bangsa, Cerita Lucu

\section{ABSTRACT}

This study aims to explain: (1) intrinsic elements; (2) the value of character education of the nation; And (3) the educational value of character of the nation. Research subjects, funny stories in Balinese literature books Language Research Institute. The object of research, intrinsic elements, the value of character education of the nation, and the value of the character education of the nation. This study uses the method of documentation of reading and writing techniques when collecting intrinsic data, the value of character education of the nation, and the value of character education of the nation. Analysis of data used: (1) data reduction; (2) description of data; And (3) conclusion / verification. The results of the study of funny stories in the literature books Bali Language Research Institute, among others: (1) intrinsic elements there are seven, such as: title, theme, character, setting background, plot, point of view, and mandate, (2) The nation is found eighteen, such as: religious, honest, tolerance, discipline, hard work, creative, independent, curiosity, friendly, peace loving, caring social, caring environment, and responsibility, (3) useful as a reflection of behavior Both in life.

Keywords: Nation Character Education, Funny Story

\section{PURWAKA}

Kasusastraan Bali pinaka silih sinunggil kasugihan Bali sane mapaiketan sareng seni lan budaya. Basa Bali pinaka sarana mababaosan ngenenin indik kahuripan krama Bali. Badudu (1975: 5) maosang kasusastraan inggih punika kria sastra sane marupa sastra gantian (basa lisan) lan sasuratan sane ngamolihang rasa sane becik.

Satua ngranjing ring kasusastraan Bali purwa. Satua madaging mustika paajah-ajahan sane mawiguna, upaminyane mustika paajah-ajahan pawatekan, kadharman, kasusilan, miwah sane tiosan. Lianan ring punika, satua madue kawigunan pinih mabuat sane ketahnyane kanggen patinget tata laksana krama Baline sajeroning nglimbakang seni sastra lan kebudayaan Bali.

Pikolih tetilikan Nurgiyantoro lan Anwar warsa 2013 wenten solas mustika paajah-ajahan pawatekan sane kapolihang, sakadi: (1) arjawa; (2) sradha bhakti; (3) tresna negara; (4) ngawi parindikan anyar; (5) seneng ngwacen; (6) tinut sesana; (7) tinut swadharma; (8) ulati palemahan; (9) teleb makarya; (10) ulati pawongan; lan (11) rasa meled uning.

Malarapan antuk kawentenan mustika paajah-ajahan pawatekan sane dahat mabuat ring satua-satua Bali kadi sane sampun kawedarang ring ajeng, panilik sarat jagi nyelehin mustika paajah-ajahan pawatekan sane wenten sajeroning satua-satua banyol ring buku kasusastraan Bali Balai Penelitian Bahasa.

Ngrereh pikobet:
tetilikan puniki,
makudang-kudang mapaiketan sareng dadalan pikobet sane sampun kabahbahang ring ajeng. Pikobet sane sampun karereh mangda mapaiketan sareng satua-satua banyol ring buku Kasusastraan Bali Balai Penelitian Bahasa. Pikobet sane sampun karereh minakadi, (1) Sapunapi wangun ring jero kria sastra sane wenten sajeroning satua-satua banyol ring buku Kasusastraan Bali Balai Penelitian Bahasa? (2) Sapunapi mustika paajah-ajahan pawatekan sajeroning satua-satua banyol ring buku Kasusastraan Bali Balai Penelitian Bahasa? (3) Sapunapi pikenoh mustika paajah-ajahan pawatekan sajeroning satua-satua banyol ring buku 
Kasusastraan Bali Balai Penelitian Bahasa? (4) Sapunapi bebaosan sajeroning satua-satua banyol ring buku Kasusastraan Bali Balai Penelitian Bahasa? lan (5) Sapunapi salampah laku saking pangawi buku satua-satua sane banyol ring Kasusastraan Bali Balai Penelitian Bahasa?

Ngwatesin pikobet: sajeroning tetilikan sane jagi kalaksanayang, panilik nureksain malih pikobet sane sampun karereh. Saking lima pikobet sane sampun katlatarang ring ajeng, panilik ngawatesin antuk tiga pikobet manten. Ring tetilikan puniki, kawentenan wangun jero kria sastra, mustika paajahajahan awatekan, lan pikenoh mustika paajah-ajahan pawatekan sajeroning satua-satua banyol ring buku Kasusastraan Bali Balai Penelitian Bahasa sane kadadosang fokus tetilikan.

Bantang pikobet tetilikan: (1) sapunapi wangun ring jero kria sastra sajeroning satua-satua banyol ring buku kasusastraan Bali Balai Penelitian Bahasa? (2) napi manten mustika paajah-ajahan pawatekan sajeroning satua-satua banyol ring buku kasusastraan Bali Balai Penelitian Bahasa? Lan (3) sapunapi pikenoh mustika paajah-ajahan pawatekan sajeroning satua-satua banyol ring buku kasusastraan Bali Balai Penelitian Bahasa?

Tetujon tetilikan: (1) prasida nlatarang wangun ring jero kria sastra sajeroning satua-satua banyol ring buku kasusastraan Bali Balai Penelitian Bahasa; (2) prasida nlatarang mustika paajah-ajahan pawatekan sajeroning satua-satua banyol ring buku kasusastraan Bali Balai Penelitian Bahasa; lan (3) prasida nlatarang pikenoh mustika paajah-ajahan pawatekan sajeroning satua-satua banyol ring buku kasusastraan Bali Balai Penelitian Bahasa.

Kawigunan pamucuk sane prasida kapolihang saking tetilikan, maguna kanggen nglimbakang widya pangweruhan basa, ngicenin pangresep paajah-ajahan indik wangun ring jero kria sastra, lan mustika paajah-ajahan pawatekan. Kawigunan panglimbak sane kapolihang: (1) majeng ring pangwacen, ngicen pangweruhan wangun ring jero kria sastra, lan mustika paajah-ajahan pawatekan; (2) majeng ring panilik lianan, kanggen pratiwimba olih panilik lianan sane madue arsa/pikayunan nglaksanayang tetilikan sane mapaiketan sareng tetilikan puniki, (3) majeng ring panilik, kanggen dasar papineh, malaksana, mabebaosan, mangda anut ring mustika paajahajahan pawatekan.

Tetilikan wangun ring jero kria sastra lan mustika paajah-ajahan pawatekan sajeroning karya sastra sujatinnyane sampun kalaksanayang olih panilik lianan. Mawit saking kawentenane punika panilik nyaihang pikolih lan tetepasan tetilikan puniki sareng tetilikan panilik lianan, sakadi: (1) tetilikan Kadek Devi Antariyani "Seseleh Wangun Intrinsik lan Kajatian Guna Sarat Paguron-guron ring Satua Pan Balang Tamak". Tatilikan puniki wantah nilikin asiki satua kemanten, inggih punika satua Pan Balang Tamak. Kapolihang pitu wangun ring jero kria sastra, lan mapikolih lima mustika paajah-ajahan pawatekan; (2) tetilikan Putu Bisma Putera Aryana "Seseleh Wangun Intrinsik lan Mustika Ajah-ajahan Pawatekan ring Sajeroning Pupulan Satua Rare "I Kentung uling Lodtungkang" Kakawian Nyoman Manda". Kapolihang pitu wangun ring jero kria sastra, dadosnyane pateh sareng tetilikan puniki. Mustika paajah-ajahan pawatekan kapolihang kutus, yening tetilikan puniki kapolihang tigalas mustika paajah-ajahan pawatekan.

Arikunto (2005:78) sepat siku-siku dahat mabuat pisan duaning prasida ngicenin pamargi lan ngwantu panilik muputang tetilikannyane. Sepat sikusiku sane kanggen, sakadi: (1) kasusastraan Bali; (2) satua; (3) wangun ring jero kria sastra; lan (4) mustika paajah-ajahan pawatekan.

Kasusastraan Bali, Suwija Ian Manda (2015:64) "kasusastraan" mateges kawentenan ajah-ajah sane luih/becik. Kasusastraan Bali inggih punika saluir ajah-ajahan/kaweruhan sane becik, pakaryan para wagmine ring 
Bali sane kasurat mabasa Bali, inggian nganggen aksara Bali lan huruf Latin.

Satua inggih punika silih sinunggil wangun kria sastra Bali sane marupa papalihan saking sastra gancaran. Satua nyritayang sapulah palih parindikan ring aab duk riin, kejatiane punika durung pastika kawentenannyene. Tinggen (2001: 19) maosang satua punika carita utawi prosa sane nenten prasida kawedarang indik kawentenannyane, utamanipun kaanggen hiburan yadiastun akeh sane nyritayang kejatian saking carita punika, madaging paplajahan susila utawi piteket lan sesimbing.

Wangun ring jero kria sastra, Tarigan (1984: 122) ngwedar baga wangun ring jero kria sastra wenten pitu, sakadi: (1) murda; (2) unteng; (3) pragina; (4) rerawatan; (5) lelintihan; (6) panampen jana; Ian (7) piteket. Mustika paajah-ajahan pawatekan, Nurgiyantoro lan Anwar (2013:384) mustika paajah-ajahan pawatekan inggih punika solah lan tata parilaksana jana sane anut ring awig (norma) sane wenten ring pakraman, sakadi spiritual, personal, sosial. Kementerian Pendidikan Nasional, baga mustika paajah-ajahan pawatekan wenten plekutus, sakadi: (1) sradha bhakti; (2) arjawa; (3) awiwahara; (4) tinut sesana; (5) teleb makria; (6) gina; (7) ngraga; (8) tatwamasi; (9) rasa meled uning; (10) dharma negara; (11) tresna negara; (12) ngajiang kawagedan; (13) masawitra; (14) metri; (15) seneng ngwacen; (16) urati pawongan; (17) urati palemahan; lan (18) tinut swadharma.

\section{KRAMANING TETILIKAN}

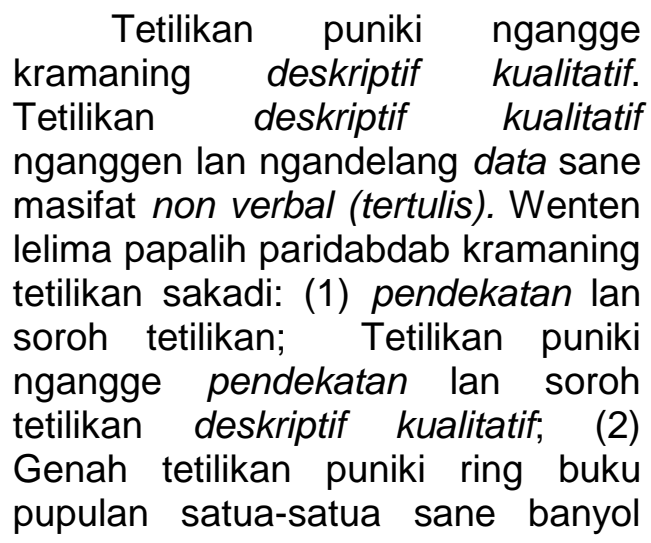

ring kasusastraan Bali, Balai Penelitian Bahasa; (3) Wit data sajeroning tetilikan puniki inggih punika satua-satua banyol ring buku Kasusastraan Bali Balai Penelitian Bahasa warsa 1976, sane kapupulang antuk I Gusti Ngurah Bagus; (4) mupulang data, Kramaning kanggen mupulang data inggih punika kramaning dokumentasi, dokumentasi ngwacen lan nyatet (teknik baca catat) kanggen, santukan data pupulan satua Bali marupa sesuratan (buku), piranti sane kanggen mupulang data inggih punika kartu data. Kartu data kanggen nyurat pikolih wangun ring jero kria sastra, lan mustika paajahajahan pawatekan; lan (5) data tureksa, pidabdab sane kalaksanayang sajeroning data tureksa sakadi ngatur, nyusun, nagingin kode, lan nlatarang data.

\section{PIKOLIH LAN TETEPASAN}

Pikolih lan tetepasan tetilikan wangun ring jero kria sastra sajeroning satua-satua banyol ring buku kasusastraan Bali Balai Penelitian Bahasa katlatarang ring sor puniki.

Murda sajeroning satua kapertama I Belog. Untengnyane kabelogan mapuara sengkala. Pragina, wenten kalih pragina sane kapanggihin sakadi, I Belog lan meme. Rerawatan, rerawatan indik genah sakadi, di sema, di jumah meten, di sember. Indik galahnyane sakadi, sedek dina anu lan nyananne. Indik kahanan sakadi, liang, sedih lan kerik-kerik.

Lelintihan ka arep. Genah sang pangawi jana kaping tiga. Piteket, sajeroning satua kapertama madue piteket mangda iraga dados jana sane wikan tur mawiguna mangda nenten kabelog-belog olih jana lian, yening iraga dados jana sane belog sinah lakar nemu sengkala. 
Murda, satua kaping kalih Satua Nang Bangsing teken I Belog. Untengnyane karma phala. Pragina, wenten solas pragina sane kapanggihin sakadi, I Belog, Nang Bangsing, Kedis Selem, I Parekan, Ida Anake Agung, I Jero Gebagan, anake di jalan, Meme, Dagang, I Jaran Berag, Anake di Badung lan anake di Sangsit.

Rerawatan indik genah sakadi, di pagehane, di umane, di jumah Nang Bangsing, di ampik, di punyan nyuhe, di puri, kaja kangin, di jabaan, di jalan, di peken, di jumah I Belog, di meten I Belog, di ampikne I Belog, di sanggah kemulan I Belog, di meten Nang Bangsing, di peken Badung lan di peken Sangsit, ba danginne, di sanggah Nang Bangsing. Indik galahnyane sakadi, buin mani semengan pesan, tengah lemeng, selid pesan, semengan, lemah, peteng. Indik kahanan sakadi, gedeg, kendel, ibuk keneh, bengong.

Lelintiha ka arep. Satua kaping kalih ngangge genah sang pangawi jana kaping tiga. Piteket, sajeroning satua kaping kalih madue piteket parilaksana becik pastika ngamolihang sane becik, yening parilaksana sane corah pastika lakar nepukin sengkala.

Murda satua bawak kaping tiga Satua Pan Belog. Untengnyane kabelogan ngamocolang angga, wenten tiga pragina sane kapanggihin sakadi, Pan Belog, Kurenan Pan Belog, lan I Dagang. Rerawatan, rerawatan indik genah sakadi, di peken, di tukad lan di jumah. Indik galahnyane sakadi, buin mani lan sedek dina anu. Indik kahanan sakadi, bengong tur sebet lan ngrengkeng.

Lelintihan ka arep. Genah sang pangawi jana kaping tiga. Piteket, sajeroning satua kaping tiga madue piteket dados jana sane wikan tur mawiguna mangda nenten dangan kabelog-belog, yening iraga dados jana sane belog sinah lakar nemu parindikan sane nenten becik utawi ngapiambengin angga miwah jana lianan.

Murda satua bawak kaping pat Satua I Nyoman Jater. Untengnyane suba belog mapi bisa. Pragina, wenten lima pragina sane kapanggihin sakadi, I Nyoman Jater, I Belenjo, Meme Wayan, Meme Nengah, Klian Pasarene. Rerawatan, rerawatan indik genah sakadi, di paon, di uma, kadaat, di jumah, kali keloda, ba dauhne, mailehan, di apit-apite, kumah meten, di pedeman, di bolongan sombahe, di pura desa, di undage lan di balen mambenge.

Indik galahnyane sakadi, saisai, bulanan, sawetara ada pukul solas, duk ento, buin manine, kali jani, lingsir, sanja, makapeteng kanti makalemah, semengan, buin telun lan ituni. Indik kahanan sakadi, med, kendel, tengkejut, kedek ngakak, keweh, engon pesan, baag-biing lan ngeling. Lelintihan ka arep. Genah sang pangawi jana kaping tiga. Piteket, sajeroning satua kaping pat madue piteket yening iraga nenten uning, sampunang kimud mataken, yening iraga nenten bisa utawi uning nanging mapi-mapi bisa sinah pacang kapetengan di jalan (nenten uning napi), lan ngamocolang dewek padidi.

Murda satua bawak kaping lima Men Muntig. Untengnyane belog kaimud ngawinang sengkala. Pragina, wenten tiga pragina sane kapanggihin sakadi, Men Muntig, Ni Muntig lan I Kayan Dokok. Rerawatan, rerawatan indik genah sakadi, di Banyuasri, di Tebusalah, di umah Ni Muntige, di paon, di teba, di pedeman jumahan meten, di tlabah, di lesunge, di grobage, di balenne, di lenggatane, di duur punapine. Indik galah sakadi, subilang peteng, mani puan, sanja, manine, tengai, semengan. Indik kahanan sakadi, lacur, demen, kendel, tusing juari, gruguh-gruguh, kedek ingkel-ingkel, tengkejut, carat- 
curut mailehan, sengitan lan utahutah.

Lelintihan ka arep. Genah sang pangawi jana kaping tiga. Piteket, sajeroning satua kaping lima madue piteket dados jana mangda nenten nulad parilaksana sane kaimud, tuah ngajumang kasugihanne dogenan.

Murda satua bawak kaping nem Satua I Clempung. Unteng ngekadaya. Pragina, wenten tiga pragina sane kapanggihin sakadi, I Clempung, I Kliang Banjar lan Men Bongkenge. Rerawatan, rerawatan indik genah sakadi, di banjar, desane dini lan ka jumahne I Clempung. Indik galahnyane sakadi, dinane buin mani, buin telun, pinih tengai, malih kalih rahina, benjang semengan. Indik kahanan sakadi, kobet, kendel lan jengah lan renget.

Lelintihan ka arep. Genah sang pangawi jana kaping tiga. Piteket, sajeroning satua kaping nem madue piteket iraga dados jana sampunang madaya sane corah, krana daya sane corah prasida ngawi parindikan sane nenten becik.

Murda satua bawak kaping pitu

Pan Balang Tamak. Untengnyane kruna daya upaya. Pragina, wenten sia pragina sane kapanggihin sakadi, Pan Balang Tamak, Anake Agung, Dusta ajaka patpat, Panyarikan desa, Sayane, Klihan desane, Krama desa, Kurenan Pan Balang Tamak, Jero Mangku. Rerawatan, rerawatan indik genah sakadi, di desa anu, ka gunung, jumah, di bengbengane, pura desa, pangkung grembeng, di alase, di punyan ketkete, di bale banjare, kabian, di sisin pekene, piasane, bale sekenem, jumah meten, ka puri, tengah bete, kori agunge, natah piasane.

Indik galahnyane sakadi, disedek dina anu, mani semengan, semengan mara tuun siap, makakali tepet, buin manine, sanja, manine mara galang kangin. Indik kahanan sakadi, sengitan, engon, yatna, jerit- jerit, sengap, mauar-uar, mwuwuhwuwuh, maselselan, bendu, tengkejut. Lelintihan ka arep. Genah sang pangawi jana kaping tiga. Piteket, sajeroning satua kaping pitu madue piteket pinaka krama desa sane becik sampun sepatutnyane iraga nginutin arah-arahan lan awigawig sane sampun kacumpuin ring pakraman.

Satua kaping kutus mamurda Satua I Belog Mantu. Untengnyane ririh madaya. Pragina, wenten lima pragina sane kapanggihin sakadi, I Belog, Ni Luh Sari, Bapan Luh Sari, Pisaga lan truna-trunane. Rerawatan, rerawatan indik genah sakadi, di bebanjarannyane, Jumahne, Kumah Luh Sari, Ka carik, di wangan Ni Luh Sarine, di natahe, di tembok paone, di paon, di dulang padaaranne, di balene, di bungbung tiing buluhe, di tengah umane, kalase, di sisin pangkunge, kayehan, di alase wayah, di piasan sanggahne, di lebuhne, ka tengah gulungan duke, di kebengan yehe, ka tengah sanggah kamulane, ka sanggahe lan tuun ka piasane, di Jalan.

Indik galahnyane sakadi, mani semengan, tengai, sanjane, ibine, suba saru mua, peteng, ibi puanne. Indik kahanan sakadi, kena kasih, dot, seneb, kedek, kedek pedih, yatna, dingin ngetor, kenyel, sebet, kendel, jerit-jerit katulung-tulung, ngeling, lega atinne, pakademi lan nyengsemang, sebet pesan. Lelintihan ka arep. Genah sang pangawi jana kaping tiga. Piteket, sajeroning satua kaping kutus madue piteket iraga dados jana mangda nenten dangan kabelogbelog.

Satua kaping sia mamurda Satua Pan Angklung Gadang. Untengnyane ngekadaya. Pragina, wenten telulas pragina sane kapanggihin sakadi, Pan Angklung Gadang, I Wayan Sebetan, I Giur, Ida Anake Agung, Kurenan Pan Angklung Gadang, Panyeroan, Jero 
Klian, I Sendi, I Tampul, I Lambang, Dagang nasi, Ancangan, Pianakne I Giur. Rerawatan, rerawatan indik genah sakadi, di desa anu, purian, ka gunung, joh ngliwat desa, di tepin desane, di bancingah, di balene di ambene,ka tukad, duur batune di tengah tukade, duur batune di luanan Ida Anake Agung, ngatebenang, di batan padagangan, di jit cicingne, jumah meten, di pabalih-balihan, di dagang nasine, ka tlabahe, di lenggatan paone, di lenggatan mambenge, di tengah glebege, kumah Pan Angkling Gadang, di banjar, di paon, di bale banjare.

Indik galahnyane sakadi, sedek dina anu, lingsir, buin puane, dauh telune, abulan, mani-puan, buin maninne, suba kone makelo, di dua bulan, tengai, uling ituni, ne malu, asasih, limang dina. Indik kahanan sakadi, caneg, egar keneh nyane, bendu, ngon pesan, dot, magretgotan, bek jeljel,gaok, uyut, jengah pesan, bengong masebeng kangen, tuara lega, masebeng jengah, engsek pesan. Lelintihan ka arep. Genah sang pangawi jana kaping tiga. Piteket, sajeroning satua kaping sia madue piteket dados jana sepatutnyane nenten demit utawi loba teken gelah timpal

Pikolih lan tetepasan tetilik mustika paajah-ajahan pawatekan satua-satua banyol ring buku kasusastraan Bali Balai Penelitian Bahasa wenten tigalas sane kapolihang, katlatarang ring sor puniki.

Sradha bhakti, kapanggihin ring satua sane mamurda Pan Belog. Data sane kapolihang sakadi puniki.

Sedek dina anu Pan Belog tundena ka peken teken kurenanne meli bebek dadua, lakar tampaha anggona banten. Krana matuanne buin maninne tutug abulan pitung dina. Kene munyinne teken Pan Belog: "Ih, bapanne, kene cai suba nawang, buin mani I bapa tutug abulan pitung dina". Sradha bhakti kacihnayang antuk parilaksana kurenanne Pan Belog matanding banten anggen tutug abulan pitung dina matuanne. Pan Belog ka pasar numbas bebek kakalih pacang tampaha kaanggen banten. Manut kawentanane ring ajeng Pan Belog sareng kurenanne nglaksanayang mustika paajahajahan pawatekan sradha bhakti, sradha bhakti prasida kacihnayang antuk parilaksana sakadi, nampah bebek anggen banten, lan ngarya sarana bebantenan/matanding.

Arjawa, kapanggihin ring satua sane mamurda Men Muntig. Data sane kapolihang sakadi puniki.

I Dokok nuturang saindik-indik dewekne kanti mising. Masaut I Dokok: "Ten, tiang nyicipin buah kacubung, malah tiang sing nyak, kaden tiang ja patuh buka buah kacubunge di desan tiange geles-geles buin pait. Ento buah kecubung memene gedegede, mara cicipin tiang laut manis, kanti pitung bungkul telah baan tiang, lantas sakit basang tiange".

Parilaksana I Kayan Dokok arjawa, kasinahang antuk I Dokok nuturang saindik-indik dewekne kantos mising. Manut ring kawentenane punika, I Kayan Dokok madue mustika paajah-ajahan pawatekan arjawa ri sajeroning satua Men Muntig puniki.

Awiwahara, kapanggihin ring satua sane mamurda Men Muntig. Data sane kapolihang sakadi puniki.

Lantas dlokina I Dokok jani ka paon, dapetanga ia negen sunduk punapi, kijep-kijep ia ditu tuara maklisik-klisikan. Lantas takonina teken matuanne.

Yadiastun parilaksana I Kayan Dokok belog-belogan dulurin budag kaimud, nanging Men Muntig lan Ni Muntig prasida masikian pinaka 
kulawarga tur prasida nerima pabinayang solah lan parilaksana I Kayan Dokok. Sumangdene prasida hidup trepti ring kawentenan pabinayan punika. Parilaksana Men Muntig lan Ni Muntig sane prasida narima pabinayan penampen, solah lan ngajinin parilaksana I Kayan Dokok sane belog-belogan dulurin kaimud, prasida kasengguh nglaksanayang mustika paajahajahan pawatekan awiwahara sajeroning kahuripan ring kulawarganyane.

Tinut sesana, kapanggihin ring satua sane mamurda Pan Balang Tamak. Data sane kapolihang sakadi puniki.

"Ih Pan Balang Tamak, mani semengan mara tuun siap, desane luas ka gunung ngalih kayu, bakal anggon menahang bale agung nyen ja kasepan, bakal kena danda". Keto araharahe teken Pan Balang Tamak. Kacrita maninne semengan mara tuun siap, luas desane makejang, nanging Pan Balang Tamak masih enu jumah padidina.

Semengan wawu tuun ayame, luas desane samian ka gunung ngrereh kayu. Parindikane punika maka cinna yening krama desane madue mustika paajah-ajahan pawatekan tinut sesana, santukan tata laksana desane sampun anut/becik sajeroning nginutin araharahan malarapan dados krama desa.

Teleb makria, kapanggihin ring satua sane mamurda I Nyoman Jater. Data sane kapolihang sakadi puniki.

Kacrita I Nyoman Jater sabilang wai magae di uma, sai-sai I Belenjo luas ka uma ngabaang nasi. Jani mara suud matekap nglantas kone ia namped-nampedang kebone, paakan di pasisi.

I Nyoman Jater sadina-dina magarapan ring carik. Parilaksana I
Nyoman Jater sane satata kukuh utawi anteng ri kala makria ring carik punika maka cihna yening I Nyoman Jater madue mustika paajah-ajahan pawatekan teleb makria sajeroning kahuripannyane.

Ngawi parindikan anyar, kapanggihin ring satua sane mamurda Men Muntig. Data sane kapolihang sakadi puniki.

Men Muntig nepung kone jani ajaka Ni Muntig. Lantas pulungpulunga tepungne amun-amun buah bunute gedednne tur mlablab aji yeh gula, adanina jaja buah bunut.

Kasinahang Men Muntig nepung sareng $\mathrm{Ni}$ Muntig. Lantas pulungpulunga tepungne amun buah bunute agengnyane tur mlablab antuk toya gendis, kawastanin jaja buah bunut. Men Muntig sareng Ni Muntig mautsaha ngawi parindikan anyar inggih punika ngawi jaja buah bunut, ipun mulungmulung tepung ngantos sekadi buah bunut wentuknyane, kawastanin jaja buah bunut. Manut kawentenane ring ajeng, Men Muntig sareng Ni Muntig madue mustika paajah-ajahan pawatekan ngawi parindikan anyar. Sida ngraga, kapanggihin ring satua sane mamurda I Belog Data sane kapolihang sakadi puniki.

Kacrita I Belog nu padidina, tusing ada anak nyakanang ia. Ban layah basangne, lantas ia ka peken meli ubi, kasela, gatep, muah ane len-lenan, ane sarwa mudah-mudah. Sasubanne betek basangne, lantas ia mulih. Jani I Belog nongos jumahne padidina, tusing ada anak ajaka ngomong.

Sajeroning satua, I Belog kari praragan, nenten wenten anak nyakanang ipun, krana memenne sampun ngalahin padem, nika sane ngawi I Belog ngraga sajeroning kahuripannyane. Parilaksana sane nenten muatang wantuan jana siosan, sakadi parilaksana I Belog kasengguh jana madue mustika paajah-ajahan pawatekan ngraga, santukan I Belog nenten satata 
nyagerang jana siosan ri kala jagi muputang pakaryan sajeroning kahuripannyane.

$$
\text { Rasa meled uning, }
$$

kapanggihin ring satua sane mamurda Satua Nang Bangsing teken I Belog. Data sane kapolihang sakadi puniki.

$\mathrm{Di}$ mulih tamiune tepukina teken Nang Bangsing, lantas ia matakon : "Ih bapa, beli, ajak makejang uli dija ne, mirib buka anake uli kundangan?" Masaut lantas ane takonina : "O, bapa uli kundangan sig I Beloge." Keto makejang patuh pasautne.

Parilaksana Nang Bangsing sane nyesed mataken majeng ring tamiune, saking dija tamiune, minab sakadi anake saking kundangan. Punika maka cihna ipun mautsaha mangda Nang Bangsing prasida uning indik kramane matamiu. Siosan ring punika, Nang Bangsing taler mautsaha nelebin mataken teken I Belog mangda prasida tatas uning ring parindikan I Belog mrasidayang ngrauhang tamiu, tur mebat-ebatan, dening I Belog punika jana lacur.

Masawitra, kapanggihin ring satua sane mamurda Satua Nang Bangsing teken I Belog. Data sane kapolihang sakadi puniki.

Ada tuturan satua anak makakasihan ajaka dadua, madan Nang Bangsing teken I Belog. Kacrita I Belog anak ia sajaan buka adanne belog pesan tur tutut. Sedek dina anu lantas ajakina I Belog masang bubu teken Nang Bangsing. Nyak kone I Belog.

Manut kawentenane ring ajeng maka cihna I Belog lan Nang Bangsing madue mustika paajahajahan pawatekan masawitra, kacihnayang antuk parilaksana sane seneng paras-paros sajeroning kahuripan utamanipun ri kala Nang Bangsing teken I Belog masang bubu. Yadiastun Nang Bansing seneng iri sareng pagelahanyane I Belog, sakewanten sida leket sang makakalih masawitra, I Belog setata asih tekening Nang Bangsing. Metri, kapanggihin ring satua sane mamurda I Belog Mantu. Data sane kapolihang sakadi puniki.

$\mathrm{Ni}$ Luh Sari ento anut madan sari, gobanne spatut, bikasne melah, magarapan ja jemet tur pakenehanne alus. Ni Luh Sari ento sayanganga pesan ben reramanne luh muani, wireh bisane ngaba awak daa jeben, seleb mangleganin keneh reramanne. Liu pesan pada trunatrunane mabudi tuara nyidayang, baan pagehne teken awak.

$\mathrm{Ni}$ Luh Sari madue mustika paajah-ajahan pawatekan metri, kacihnayang antuk $\mathrm{Ni}$ Luh Sari anut mawasta sari, parasnyane sapatut, parilaksananyane melah, magarapan jemet tur manah ipune alus. Ni Luh Sari sayanganga pesan antuk reramanne lanang istri, sawireh prasida makta raga daa jeben, seleb mangleganin manah rerama ipune. Akeh pesan pada trunatrunane mabudi nenten mrasidayang, santukan pageh ipune. Parilaksana $\mathrm{Ni}$ Luh Sari dahat becik pisan prasida ngawi karahayuan ring kahuripan.

Urati pawongan, kapanggihin ring satua sane mamurda I Nyoman Jater. Data sane kapolihang sakadi puniki.

Duk ento nujuang ada sekaa jaring tokal nyaring bano, mara ngasisiang jukung lantas I Nyoman Jater milu nulungin ngedengang jukung muah ngosongang ngaba ka daat. Disubanne pada suud ngosong jukung, lantas I Nyoman Jater upahina kone bano abesik.

Parilaksana urati pawongan prasida kacihnyang antuk parilaksana ngicen wantuan, sakadi I Nyoman Jater milu nulungin ngedengang jukung muah ngosongang makta ka daat. Dados jana sampun sepatutnyane iraga saling wantu, ngicen wantuan majeng ring jana tiosan. Punika maka cihna yening I Nyoman Jater madue mustika paajahajahan pawatekan urati pawongan.

Urati palemahan, kapanggihin ring satua sane mamurda I Belog 
Mantu. Data sane kapolihang sakadi puniki.

Suba ia ngomong keto, buin mamunyi bapan Luh Sari : "Belog, mani jalan ngalih duk kalase, barengin bapa. Bakal anggon raab sanggahe. Apa onyangan suba uug". Masaut I Belog: "Nah, bapa".

Kawentenan bapan Luh Sari sane ngalih duk kalase, pacang kaanggen raab mrajan krana mrajan ipune sampun rusak, maka cihna bapan Luh Sari taler madue mustika paajah-ajahan pawatekan urati palemahan malarapan antuk urati pacang menahin mrajan ipun sane sampun rusak.

Tinut swadharma, kapanggihin ring satua sane mamurda Pan Angklung Gadang. Data sane kapolihang sakadi puniki.

Kacrita jani krama banjare suba pepek masangkepan, ditu laut jero klian ngujangang kramane ne mutrain : "I Sendi." "Tiang. Naur nika". Sret ngembud satakan laut mayah. "I Tampul : "Tiang. Mangkin, mangkin, tiang naur". Krasakkrisik ngagah bebuntilan nglaut mayah.

Krama desane sekadi I Sendi Ian I Tampul sampun nyihnayang parilaksana tinut swadharma. Kacihnayang antuk ipun eling ring swadarma naur utang. Yening iraga madue utang, sampun sepatutnyana utang punika kataur. Manut ring kawentenane punika, Krama desane sekadi I Sendi lan I Tampul madue mustika paajah-ajahan pawatekan tinut swadharma, kacihnayang antuk parilaksana eling ring swadharma naur utang.

\footnotetext{
Pikolih lan tetepasan tetilikan pikenoh mustika paajah-ajahan pawatekan satua-satua banyol ring buku kasusastraan Bali Balai Penelitian Bahasa sane kapolihang, katlatarang ring sor puniki.
}

Mustika paajah-ajahan pawatekan prasida kaanggen sesuluh sajeroning kahuripan jana. Mustika paajah-ajahan pawatekan mangda karesepang tur kalaksanayang ring kahuripan sarahina, mangda sayan eling ring sesana dados jana. Yening para jana prasida nelebin mustika paajah-ajahan sane luwih pastika wangsa puniki prasida nglimbak. Paajah-ajahan pawatekan prasida ngawi panagara sayan kukuh lan manggihin karahayuan yening parajanane sida ngukuhang lan nglaksanayang paajahajahan pawatekan sane mabuat pisan sajeroning kaanggen panutan lan pratiwimba yening jagi mapineh, mabaos lan maparilaksana, mangda prasida manggihin kasutreptian ring jagate

Implikasi ring tatilikan puniki inggih punika sakadi sane sampun ketah kauningin, suksman satua madaging lan ngutamayang makudang-kudang guna sarat, norma-norma, wiadin hukum karma. Punika taler pikolih mustika paajahajahan pawatekan lan pikenoh sane kapanggih ring tetilikan puniki prasida kaanggen sinalih tunggil wit paajah-ajahan pawatekan majeng ring parajana lan kanggen nabdabin parilaksananyane. Pikolih tetilikan puniki dahat mabuat katelebin lan prasida anggen sesuluh, santukan akeh pikenoh sane kapanggih sajeroning satua-satua banyol puniki. Pamekas nyane dahat mawiguna majeng sang sane ngwacen.

\section{PAMUPUT}

Manut pikolih lan tetepasan tetilikan sane sampun katlatarang, prasida kacutetang wangun ring jero kria sastra sajeroning satua-satua banyol ring buku kasusastraan Bali Balai Penelitian Bahasa wenten 7 sakadi: (1) murda; (2) unteng; (3) pragina; (4) rerawatan; (5) lelintihan; (6) genah sang pangawi; lan (7) piteket.

Mustika paajah-ajahan pawatekan sajeroning satua-satua banyol ring buku kasusastraan Bali 
Balai Penelitian Bahasa wenten 13 sakadi: (1) sradha bhakti; (2) arjawa; (3) awiwahara; (4) tinut sesana; (5) teleb makria; (6) ngawi parindikan anyar; (7) ngraga; (8) rasa meled uning; (9) masawitra; (10) metri; (11) urati pawongan; (12) urati palemahan; Ian (13) tinut swadharma.

Pikenoh mustika paajahajahan pawatekan sane wenten sajeroning satua-satua banyol ring buku kesusastraan Bali Balai Penelitian Bahasa puniki inggih punika Mustika paajah-ajahan pawatekan prasida kaanggen sesuluh sajeroning kahuripan jana. Mustika paajah-ajahan pawatekan mangda karesepang tur kalaksanayang ring kahuripan sarahina, mangda sayan eling ring sesana dados jana

Piteket kawedarang majeng ring: (1) sisia/mahasisia mangda nguratiang satua Bali, madaging kaluihan mustika paajah-ajahan pawatekan sane patut kanggen sesuluh lan kalaksanayang; (2) sang sane ngwacen mangda sayan urati, lan mautsaha nglestariang tetamian panglingsire indik satua Bali. Satua madaging kaluihan, pangresep wangun ring jero kria sastra, mustika paajah-ajahan pawatekan, lan pikenoh mustika paajah-ajahan pawatekan; (3) panilik lianan mangda sida nglimbakang malih daging tetilikan sane mapaiketan sareng basa Bali, utamannyane wangun ring jero kria sastra, mustika paajah-ajahan pawatekan lan pikenoh mustika paajah-ajahan pawatekan.

\section{KAPUSTAKAAN}

Badudu, J. S. 1975. Sari Kesusastraan Indonesia. Bandung: Pustaka Prima.

Bagus, I Gusti Ngurah. 1976. Satuasatua sane Banyol ring Kasusastraan Bali. Singaraja: Balai Penelitian Bahasa.

Devi Antariyani, Kadek. 2015. Seseleh Wangun Intrinsik lan Kejatian Guna Sarat Paguronguron ring Satua Pan Balang Tamak. Skripsi (nenten kamijilang). Jurusan Pendidikan Bahasa Bali, FBS, Undiksha Singaraja.

Nurgiyantoro, Burham. 2005. Teori Pengkajian Fiksi. Yogyakarta: Gadjah Mada University Press. Burhan Ian Anwar Efendi. 2013. "Prioritas Penentuan NilaiPendidikan Karakter dalam Pembelajaran Sastra Remaja". Cakrawala Pendidikan Jurnal, Volume XXXII, Nomor 3 (kaca. 113124).

Suwija, I Nyoman lan I Gede Manda. 2011. Widia Sari Basa Lan Sastra Bali I untuk SMA/SMK. Denpasar: Sri Rama.

Tarigan, Henry Guntur. 1984. Prinsip-prinsipDasar Sastra. Bandung: Angkasa.

Tinggen, I Nengah. 2001. Kumpulan Buku Satua. Singaraja: Universitas Pendidikan Ganesha.

Aryana, Putu Bisma Putera. 2016. Seseleh Wangun Intrinsik lan Mustika Ajah-ajahan Pawatekan ring Sajeroning Pupulan Satua Rare "I Kentung Uling Lodtungkang"Kakawian Nyoman Manda. Skripsi (nenten kamijilang). Jurusan Pendidikan Bahasa Bali, FBS, Undiksha Singaraja 